\title{
Contact Force Measurement of Instruments for Force-Feedback on a Surgical Robot: Acceleration Force Cancellations Based on Acceleration Sensor Readings
}

\author{
Shigeyuki Shimachi ${ }^{1}$, Fumie Kameyama ${ }^{1}$, Yoshihide Hakozaki ${ }^{1}$, \\ and Yasunori Fujiwara ${ }^{2}$ \\ ${ }^{1}$ Iwate University, 4-3-5 Ueda, Morioka, Japan, 020-8551 \\ shimachi@iwate-u.ac.jp \\ ${ }^{2}$ Ichinoseki National College of Technology, Hagisho, Ichinoseki, Japan, 021-8551
}

\begin{abstract}
For delicate operations conducted using surgical robot systems, surgeons need to receive information regarding the contact forces on the tips of surgical instruments. For the detection of this contact force, one of the authors previously proposed a new method, called the overcoat method, in which the instrument is supported by sensors positioned on the overcoat pipe. This method requires cancellation of the acceleration forces of the instrument/holder attached to the overcoat sensor. In the present report, the authors attempt to use acceleration sensors to obtain the acceleration forces of the instrument/holder. The new cancellation method provides a force-detection accuracy of approximately $0.05-0.1 \mathrm{~N}$ for a dynamic response range of up to approximately $20 \mathrm{~Hz}$, compared to approximately $1 \mathrm{~Hz}$, which was achieved by using acceleration forces based on the theoretical robot motion.
\end{abstract}

\section{Introduction}

In laparoscopic surgery, a surgeon operates using specially designed instruments through ports formed in the patient's abdomen. This technique reduces surgical damages to the patient's body and results in a shortened recovery period. However, the surgeon is significantly constrained with respect to the loss of direct visual information and manual operations. Surgical robot systems such as the da Vinci system and the Zeus system have substantially reduced these constraints. However, at present, haptic feedback is not provided by these systems. Although surgeons are able to view tissue deformation as a measure of external force, this type of visual compensation is limited to elastic materials and is not suitable for bone structure or suture materials.

A great number of studies have been conducted, though the references are omitted here, in order to investigate 1) the tactile force, 2) the grasping force of the forceps, and 3) the tip end force of the instrument, in combination with a) the effect of force feedback on the surgeon's skills as well as the development of b) force sensors, c) a man-machine interface and d) bilateral controls. The present report concerns the development of $b$ ) force sensor equipment for use on a slave robot for sensing the 3) tip end force of the instrument.

Taylor [1] pointed out that the friction force between the instrument and the trocar may limit sensitivity of the external force detection. Madhani [2] reported a vital 
method for estimating the tip force from the motor torques that drive a multipledegree-of-freedom forceps. Although this system was tested on a master slave system, the force feedback was reported to give the operator annoyance. Seibold [3] installed a six-axis force sensor to the end of a forceps, but reported that precise sensing is difficult with respect to forces in the direction of the shaft, which include the pulling force for the gripping jaws of the forceps.

One of authors [4] proposed a new method, called the overcoat method, in which the instrument is supported by force sensors that are located on the inner face of the overcoat pipe, as shown in Fig.1. The overcoat sensing system accepts most types of instruments. In addition, this system allows measurement of the external force acting on the tip of the surgical instrument attached to the slave robot in laparoscopic robotic surgery and is free from the frictional forces generated on the trocar.

The main concern regarding the overcoat method is the construction of the force sensors. In our first attempt [4], three-axis force sensors were placed on the outside of the abdomen and were driven at semi-static speed. This structure provided only a short sensing range of force magnitude. In our second stage attempts [5],[6], in order to provide higher speed of the instrument motion, the acceleration force derived from the instrument mass was compensated with the acceleration force due to the robot motion. However, the obtained dynamic response was only up to approximately $1 \mathrm{~Hz}$. This poor response is a result of the robot arm motion. Robot has inherently character frequency. Main character frequency of industrial robot is around from several $\mathrm{Hz}$ to $20 \mathrm{~Hz}$. The robot used as test equipment has the character frequency of approximately $7 \mathrm{~Hz}$. Low-pass filtering of $2 \mathrm{~Hz}$ yields a measuring system response of around $1 \mathrm{~Hz}$.

For detecting delicate changes of contact force and for applying the overcoat method to a robot that has such rigidity as that of industrial robot, we have to improve the dynamic response of the force measurement system.

Recently, small acceleration sensors have become available. The present report describes an attempt to improve the response characteristic of the measurement system by means of direct measurement of the acceleration of the instrument mass for compensating the acceleration force.

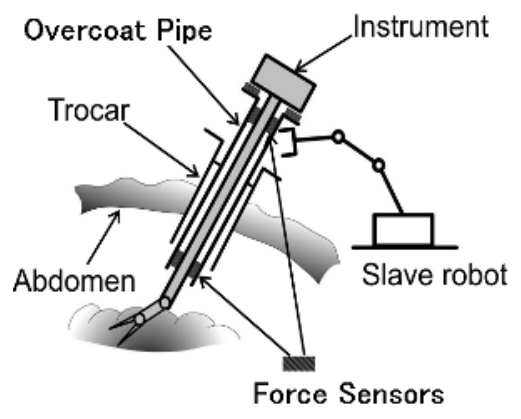

Fig. 1. Basic principle of overcoat method. Force sensors support instrument.

\section{Theory of Force Measurement}

Feedback Force Components. The goal of the present study is to provide force feedback to the fingertips of the surgeon. So, three orthogonal force components acting on the tip end of the instrument are measured herein.

Outline of the Overcoat Method. Fig.1 shows the basic principle of the overcoat method. A number of sensors that are installed inside the overcoat pipe support the 
instrument/holder that contains the instrument, the driving mechanisms and the holders for the instrument. The overcoat pipe is inserted into a trocar and is handled by a slave robot hand. In actual construction, the instrument shaft is inserted into an inner pipe and the force sensors are arranged between the inner pipe and the overcoat pipe. The mass of the instrument/holder and its acceleration make up the acceleration force. If the acceleration force can be subtracted from the force measured by the overcoat force sensors, then the force acting on the tip end of the instrument can be detected.

\section{Coordinate System and Force Balance.}

Fig.2 shows the shaft of the instrument and the $\mathrm{X}, \mathrm{Y}, \mathrm{Z}$ coordinate system fixed on the ground. As shown in Fig.2, in the present report, the shaft direction of the instrument will be oscillated. For the expression of forces, let us introduce new coordinate axes and fix them to the instrument so as that the $\mathrm{z}$ axis coincides with the shaft of the in-

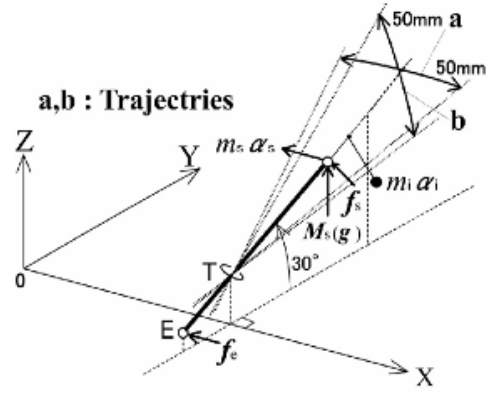

Fig. 2. Test trajectories for point "R" strument, the $\mathrm{x}$ axis is set parallel to the ground plane at the starting position of the instrument and the $\mathrm{x}, \mathrm{y}$ and $\mathrm{z}$ coordinates form an orthogonal coordinate system, as shown in Fig.5.

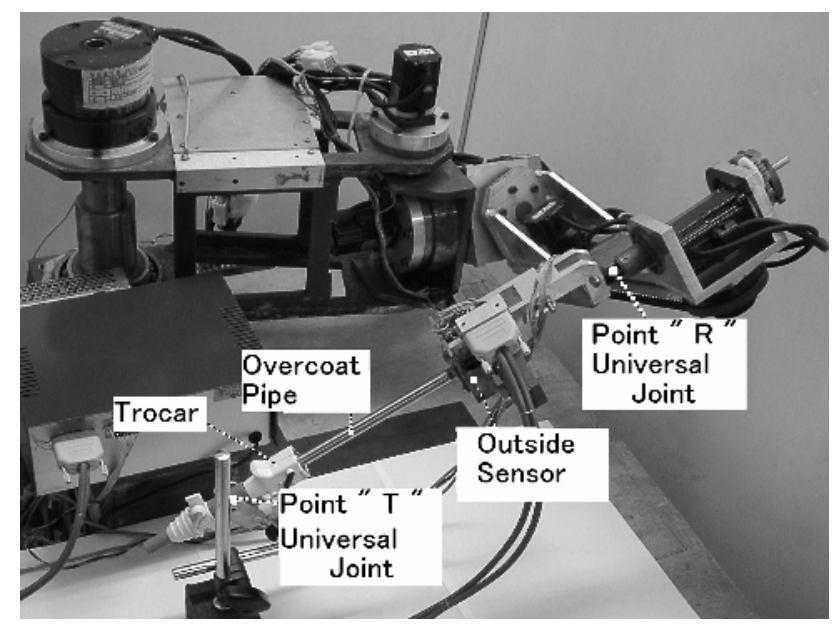

Fig. 3. Test setup of the overcoat sensing system

Let us define the following: $f_{e}\left(f_{x}, f_{y}, f_{z}\right)$ is the detected force that acts on the tip end of the instrument, $\boldsymbol{f}_{s}\left(f_{s x} f_{s y} f_{s z}\right)$ is the force sensed by the overcoat sensors, $m_{i}$ is the mass of the instrument/holder, $\boldsymbol{\alpha}_{i}$ is the motional acceleration of the center of gravity of the instrument/holder, $m_{s}$ is the mass of the overcoat sensor frame, $\boldsymbol{\alpha}_{s}$ is the motional acceleration of the gravity center of the overcoat sensor frame, and $\boldsymbol{g}$ is the gravitational acceleration. The force balance of the instrument/holder is as follows: 


$$
\begin{aligned}
& \boldsymbol{f}_{\boldsymbol{e}}+\boldsymbol{f}_{s}+m_{i}\left(\boldsymbol{\alpha}_{i}+\boldsymbol{g}\right)+m_{s}\left(\boldsymbol{\alpha}_{s}+\boldsymbol{g}\right)=0, \\
& \boldsymbol{a}_{i}=\boldsymbol{\alpha}_{\boldsymbol{i}}+\boldsymbol{g}, \boldsymbol{a}_{s}=\boldsymbol{\alpha}_{s}+\boldsymbol{g} .
\end{aligned}
$$

Here, the accelerations $\boldsymbol{a}_{i}\left(a_{i x}, a_{i y}, a_{i z}\right)$ and $\boldsymbol{a}_{s}\left(a_{s x}, a_{s y}, a_{s z}\right)$ are to be measured directly using acceleration sensors.

\section{Experimental System}

Experimental System. Fig.3 shows anoverview of the experimental system. The system is composed of a six-axis slave robot, the overcoat sensor, an instrument/holder and a trocar (commercial item) supported by a universal joint mechanism. The base frame of the overcoat sensor is attached to the robot hand through a universal joint for adjusting miss-alignment between the overcoat sensor pipe axis and the robot hand rotational axis.

Overcoat Sensor. The overcoat sensor consists of three stainless pipes: an inner pipe, through which the instrument is inserted, a force sensor pipe, to which deflectable beams are attached for strain gauges, and an outer pipe (overcoat pipe), which covers the sensor pipe. The inner pipe has an inner diameter of $5.5 \mathrm{~mm}$, and the outer pipe has an outer diameter of 10 mm. Fig.4 (top photograph) shows the inner part of the sensor pipe. Here, the outer pipe is removed. The 0.5 -mm-thick sensor pipe has two sets of parallel deflection beams for the force component $f_{s x i}$ and that for the $f_{s y i}$, as well as a deflection beam

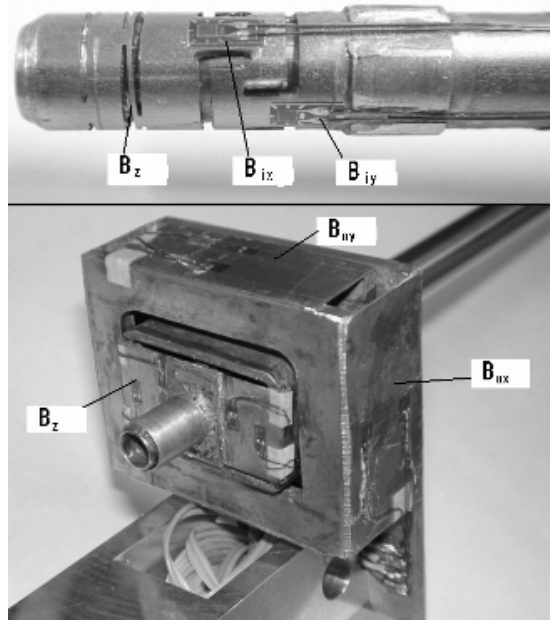

Fig. 4. Overcoat sensor. (top); inner part that is set on the inside $B_{z}$ : bending beams for $\mathrm{x}, \mathrm{y}$ and $\mathrm{z}$ forces, of abdomen, $\mathrm{B}_{\mathrm{ix}}, \mathrm{B}_{\mathrm{iy}}$, $\mathrm{B}_{\mathrm{z}}$ : bending beams for $\mathrm{x}, \mathrm{y}$ and $\mathrm{z}$ directional forces, (bottom); outer part, $\mathrm{B}_{\mathrm{ox}}, \mathrm{B}_{\mathrm{oy}}$, bending beams for $\mathrm{x}, \mathrm{y}$ and $\mathrm{z}$ forces. structure for allowing displacement by $f_{s z}$ in the shaft direction. Fig.3 (bottom photograph) shows the outside sensor part that is placed on the outside of the abdomen. This sensor part has three sensor components. Each sensor component has a set of two parallel bending plates for sensing each force component. The three sensor components for the $f_{\text {sxo }}, f_{\text {syo }}$ and $f_{s z}$ directional forces are stacked one-by-one inside the sensor in a box shape.

The overcoat sensor has five outputs $f_{s x i}, f_{s y i}, f_{s x o}, f_{s y o}$ and $f_{s z}$. The $\mathrm{x}$ and y components are summed as $f_{s x}=f_{s x i}+f_{s x o}$ and $f_{s y}=f_{s y i}+f_{\text {syo }}$ using a computer. The masses of the outer sensor frames, onto which parallel bending plates are fixed, are $120.4 \mathrm{~g}$ for $f_{s x}, 85.7 \mathrm{~g}$ for $f_{s y}$, and $10.7 \mathrm{~g}$ for $f_{s z}$, respectively. The sensors have the linearity approximately $5 \%$ for $10 \mathrm{~N}$ and the resolution smaller than around $0.02 \mathrm{~N}$, as low pass filtered signals. 
Trocar and Instrument/Holder. A commercially available trocar for an instrument shaft diameter of $10 \mathrm{~mm}$ was attached to a universal joint mechanism. The design of instrument/holder mechanisms is one of the future subjects. To test the overcoat method, we have to assume the instrument/holder mass. So, the authors made a simple holder in which a commercial and hand operate forceps is installed and the openclose motion of the jaws of forceps is driven by a geared motor (back side of the plate) that has larger power than the required one, as shown in Fig.5. The total weight of the trial instrument/holder is approximately $350 \mathrm{~g}$. So, the authors attached a model weight $(400 \mathrm{~g} \cong 350 \mathrm{~g})$ to the inner pipe, as shown in Fig. 6. Authors expect now that the weight of instrument/holder may have around 500g. In this case, $\boldsymbol{a}_{\boldsymbol{s}}$ and $\boldsymbol{a}_{\boldsymbol{i}}$ become equal (, and it will be expressed as $\boldsymbol{a}_{s}\left(a_{s x}, a_{s y}, a_{s z}\right)$ ).

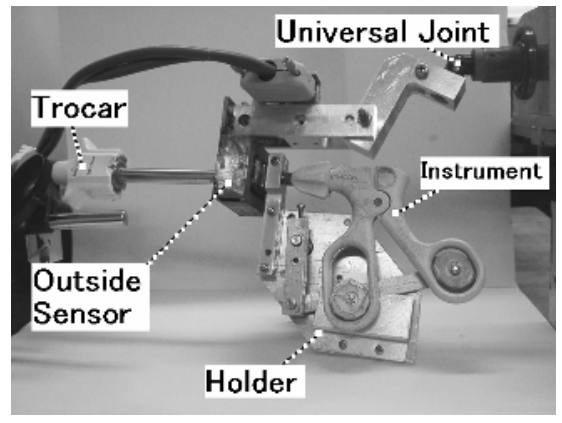

Fig. 5. An example of the instrument/holder setup

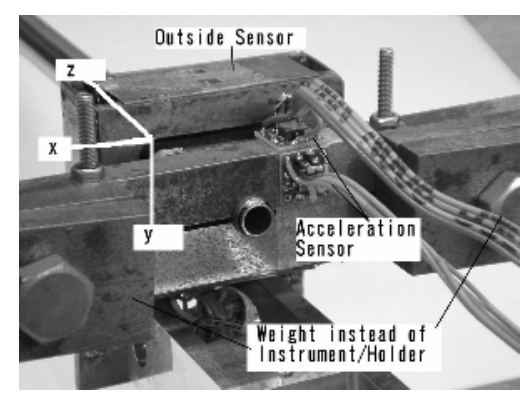

Fig. 6. Acceleration sensors and weight that represents the instrument/holder

Acceleration Sensor and Low Pass Filters. A two-directional acceleration sensor ADXL202E (Analog Devices Co.) was used as an acceleration sensor. The sensor has the outer dimensions of approximately $5 \mathrm{~mm} \times 5 \mathrm{~mm} \times 2 \mathrm{~mm}$ and a cross-axis sensitivity of approximately $2 \%$. For three-directional sensing, two sensors are arranged as shown in Fig.6.

The sensor outputs were passed through Low-Pass Filter (LPF) system, as shown in Fig. 7. The LPF generally gives a phase sift to the signal. Calculation must be performed for several output signals. So, we used filters that have almost the same characteristic for each of the signal passes. The acceleration sensor has two types of output signal; analog type and pulse width modulation (PWM) type. PWM type was selected in consideration of noise mixing in signal transmission. In order to obtain a smooth analog signal, a 2nd-order

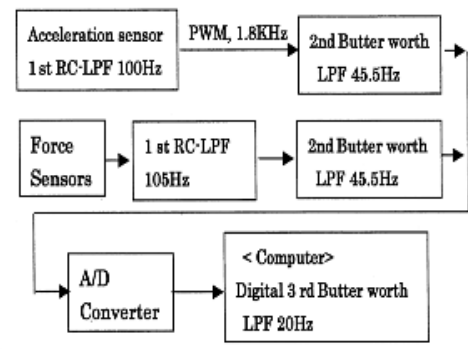

Fig. 7. Low-pass filter system Butterworth LPF (approximately $50 \mathrm{~Hz}$ ) was used, as shown in Fig. 7. The LPF for the outputs of the force sensors were adjusted to those of the acceleration sensors, in 
order to obtain almost the same characteristic. The above-mentioned values depend on the nominal values of the R C electric parts, and so have the same accuracy.

Slave Robot. The slave robot is a type of six-axis serial linkage. The rotation rigidity around the first axis of the robot arm is low, and its character frequency is approximately $7-8 \mathrm{~Hz}$ which is in the character frequency range of typical industrial robot.

\section{Experimental Results}

Experimental Trajectories of the Robot Hand. The robot hand point " $\mathrm{R}$ " was driven sinusoidally in two trajectories "a" and "b", as shown in Fig.2. The starting position of the instrument shaft is inclined $30^{\circ}$ from $\mathrm{X}-\mathrm{Y}$ plane and is parallel to the $\mathrm{Z}-\mathrm{Y}$ plane. Trajectory "a" exists on a plane parallel to the Z-Y plane and has an oscillation of ( $u \sin (2 \pi f t), f$ : frequency) for an amplitude $(u=50 \mathrm{~mm}$ in circular arc) around the trocar point "T". Trajectory " $b$ " has the same oscillation as that of trajectory "a", but the swing axis exists on a plane parallel to the Z-Y plane. We can easily observe the motional acceleration effect in trajectory "a" and the gravitational acceleration effect in the trajectory " $b$ ".

Data Acquisition and Procession. All of the input signals were reset to zero at the starting position of the trajectories. The force and acceleration by each sensor were measured three times for both trajectories "a" and "b" at a speed $0.5 \mathrm{~Hz}$ without loading the tip end of the instrument, i.e., $f_{x}=f_{y}=f_{z}=0$.

In canceling the acceleration forces, we have to consider the cross-axis sensitivity of the acceleration sensor. Therefore, the overcoat force sensor outputs $f_{s x}, f_{s y}$, and $f_{s z}$ were cancelled through the following expression:

$$
\begin{gathered}
\left(f_{x}, f_{y}, f_{z}\right)^{\mathrm{T}}=\left(f_{s x}, f_{s y}, f_{s z}\right)^{\mathrm{T}}-\mathrm{K}\left(\left(a_{x}, a_{y}, a_{z}\right)^{\mathrm{T}}-\mathrm{c}^{\mathrm{T}}\right), \\
\mathrm{K}=\left(\begin{array}{ccc}
-0.6360 & 0.0626 & -0.0182 \\
0.0242 & -0.4120 & 0.0249 \\
0.0081 & 0.0651 & -0.4504
\end{array}\right), \quad \mathrm{c}=(0.0021,-0.0166,0.0035) .
\end{gathered}
$$

Here, the diagonal coefficients of the matrix " $\mathrm{K}$ " correspond to the masses of each directional sensor, and the other values show the effects of the cross-axis sensitivity. The constant "c" corresponds to the offset of the acceleration sensor outputs.

The constants " $\mathrm{K}$ " and "c" are calculated using a regression for each force direction $\mathrm{x}, \mathrm{y}$ and $\mathrm{z}$, for each data corresponding to the three measurements for both trajectories "a" and "b", under the condition $f_{x}=f_{y}=f_{z}=0$. The 18 sets of constants are averaged to obtain the constants shown in Eq. (2).

The diagonal constant referring to the $\mathrm{x}$ direction on the matrix " $\mathrm{K}$ " shows that the mass in the $\mathrm{x}$ direction is approximately $0.636 \mathrm{Kg}$. This value differs from the measured mass, i.e., $(0.400+0.120=0.520 \mathrm{Kg} / 0.636 \mathrm{Kg})$, and the same method yields $0.486 \mathrm{Kg} / 0.412 \mathrm{Kg}$ for $\mathrm{y}$ and $0.411 \mathrm{Kg} / 0.450 \mathrm{Kg}$ for $\mathrm{z}$, respectively. These values exceed the expected accuracy, which is smaller than $10 \%$. Poor accuracy with regard to the $\mathrm{x}$ directional data, will also be shown later. 


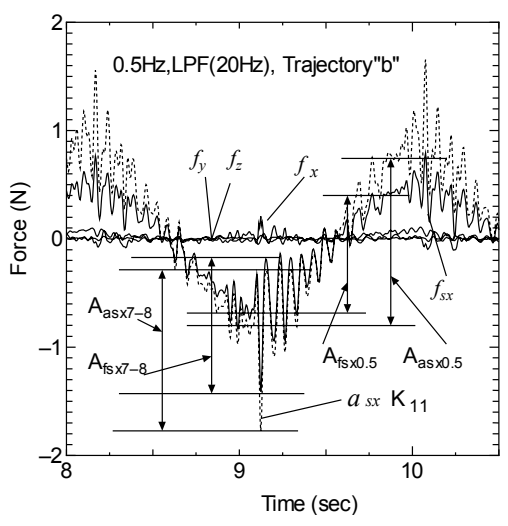

Fig. 8. Example of force cancellation on trajectory "b". $f_{s x}$; force sensor output, $a_{s x}$; acceleration sensor output, $f_{x}, f_{y}, f_{z}$; detected forces, $\mathrm{A}_{\mathrm{fsx}}, \mathrm{A}_{\text {asx }}$ : amplitude of $f_{s x}, a_{s x}$ for frequency subscript $7-8: 7-8 \mathrm{~Hz}$, subscript $0.5: 0.5 \mathrm{~Hz}$, $\mathrm{K}_{11}: 11$ component of matrix $\mathrm{K}$.

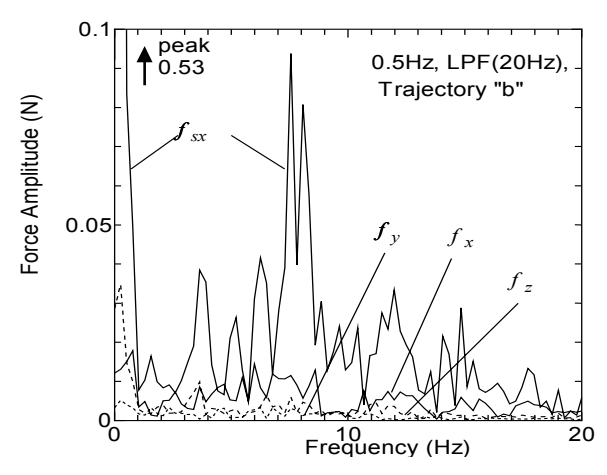

Fig. 9. Force amplitude/frequency analysis by FFT for the data shown in Fig.8

Measurement Errors. As the worst case, which shows the largest error in the detected forces, Fig. 8 shows the force component $f_{s x}$ for trajectory "b" and the detected forces $f_{x}, f_{y}$, and $f_{z}$ that should be zero. Fig.9 shows the results of FFT analysis for the same data of the forces $f_{s x}, f_{x}, f_{y}$ and $f_{z}$. Fig.9 shows that the amplitude of the signal $f_{s x}$ in the frequency ranges of up to approximately $20 \mathrm{~Hz}$ is almost canceled by the acceleration forces.

As shown in Fig.8, the accelerationsensor output $a_{s x}$ follows the force $f_{s x}$ without phase lag of the signal, even though the robot and with large amplitude after approximately 9 seconds. However, the large amplitude of the resonance frequency of 7-8 Hz gives the largest error for the detected force $f_{x}$. In Fig. 8 , the four arrows indicate two types of amplitude $\mathrm{A}_{\mathrm{fsx}}, \mathrm{A}_{\mathrm{asx}}$ for the force $f_{s x}$ and the acceleration $a_{s x}$, and for frequency $0.5 \mathrm{~Hz}$ and $7-8 \mathrm{~Hz}$, respectively. The ratio (amplitude $\mathrm{A}_{\mathrm{fsx} 7-8}$ of the force $f_{x}$ at a frequency 7-8 Hz) /(amplitude $\mathrm{A}_{\mathrm{fsx} 0.5}$ of the force $f_{x}$ at a frequency of $0.5 \mathrm{~Hz}$ ) is approximately 1.3 times of that

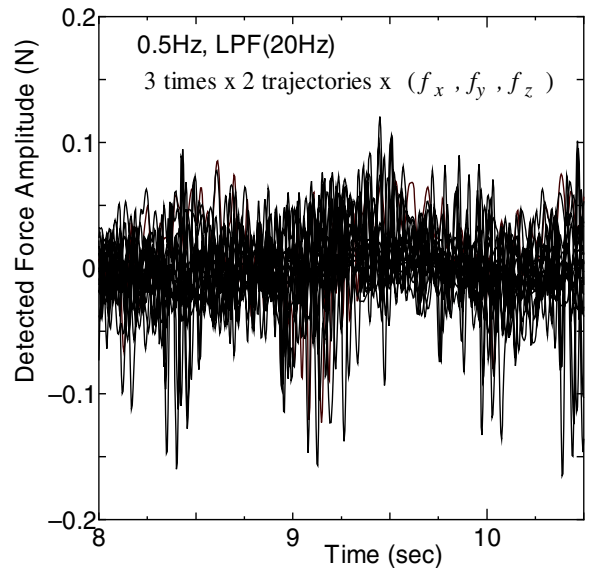

Fig. 10. Detected force amplitude for hand starts oscillating severely no-contact condition, i.e. measurement error $\left(\mathrm{A}_{\text {asx } 7-8} / \mathrm{A}_{\text {asx } 0.5}\right)$ of the acceleration $a_{s x}$. This makes the detected force errors large. This is a future problem that must be solved in order to improve fine force detection. 
Fig.10 shows the detected forces $f_{x}, f_{y}$, and $f_{z}$ for trajectories "a" and "b", and for three measurements. The amplitude of the detected force for no-contact condition corresponds to the measurement error of the system. The amplitude of the force is in the range of approximately $0.05-0.1 \mathrm{~N}$, for every kind of the forces.

\section{Conclusion}

In order to feed back the contact force of a surgical instrument to the surgeon in a laparoscopic surgical robot system, the overcoat method has been previously proposed by one of authors. This method requires cancellation of the acceleration force of the instrument/holder. In the present study, the authors attempted to use acceleration sensors to estimate the acceleration forces of the instrument/holder, and the following results were obtained. The cancellations performed using the acceleration sensors can provide a force-detection accuracy of approximately $0.05-0.1 \mathrm{~N}$ for a dynamic range of up to approximately $20 \mathrm{~Hz}$.

The cancellation method examined herein improved the dynamic response range to $20 \mathrm{~Hz}$ from $1 \mathrm{~Hz}$ by using robot motion for calculating the acceleration of the instrument/holder.

\section{References}

1. R. H. Taylor, J. Funda, B. Eldridge, S. Gomory, K. Gruben, D. LaRose, M. Talamini, L. Kavoussi, and J. Anderson:A Telerobotic Assistant for Laparoscopic Surgery,IEEE Engineering in Medicine and Biology, Vol. 14, No. 3, pp. 279-288 (1995).

2. A. J. Madhani, G. Niemeyer and J. K. Salisbury Jr.,The Black Falcon: A Teleoperated Surgical Instrument for Minimally Invasive Surgery, Proc. IEEE Int. Conf. Intelligent Robots and Systems, pp.936-944, 1998.

3. Ulrich Seibold, Gerd Hirzinger, A 6-Axis Force/Torque Sensor Design for Haptic feedback in minimally invasive robotic surgery, Proc. of the 2nd VDE World Microtechnologies Congress, October, 2003

4. S.Shimachi, A. Oguni, and A. Hashimoto:Assist Robot System for Laparoscopic Surgery (Feedback Performance on the Forceps Load), Proc. 36th JSME Spring Annual Meeting at Tohoku Branch, pp. 88-89 (2001) (in Japanese).

5. S.Shimachi,Y.Hakkozaki,T.Tada,Y.Fujiwara, Measurement of force acting on surgical instrument for force-feedback to master robot console, CARS2003 (Elsevier), pp538-546

6. S.Shimachi, Y.Fujiwara, Y.Hakkozaki, New sensing method of force acting on instrument for laparoscopic robot surgery, CARS2004 (Elsevier), pp775-780 